\title{
Orexin A/Hypocretin-1 Selectively Promotes Motivation for Positive Reinforcers
}

\author{
Stephanie L. Borgland, ${ }^{1,5}$ Shao-Ju Chang, ${ }^{1}$ M. Scott Bowers, ${ }^{1}$ Jennifer L. Thompson, ${ }^{5}$ Nicole Vittoz, ${ }^{3}$ Stan B. Floresco, ${ }^{4}$ \\ Jonathan Chou, ${ }^{1}$ Billy T. Chen, ${ }^{1}$ and Antonello Bonci ${ }^{1,2}$ \\ ${ }^{1}$ Ernest Gallo Clinic and Research Center, Department of Neurology, and ${ }^{2}$ Wheeler Center for the Neurobiology of Addiction, University of California, San \\ Francisco, San Francisco, California 94110, ${ }^{3}$ Department of Psychiatry, The University of British Columbia, Vancouver, British Columbia V6T 2B5, Canada, \\ and Departments of ${ }^{4}$ Psychology and ${ }^{5}$ Anesthesiology, Pharmacology, and Therapeutics, The University of British Columbia, Vancouver, British \\ Columbia V6T 1Z3, Canada
}

Orexin A/hypocretin-1 (oxA/hcrt-1) is known to be a modulator of dopamine-dependent neuronal activity and behaviors. However, the role of this system in driving motivated behaviors remains poorly understood. Here, we show that orexin/hypocretin receptor-1 (ox/hcrt1R) signaling is important for motivation for highly salient, positive reinforcement. Blockade of ox/hcrt-1R selectively reduced work to self-administer cocaine or high fat food pellets. Moreover, oxA/hcrt-1 strengthened presynaptic glutamatergic inputs to the ventral tegmental area (VTA) only in cocaine or high fat self-administering rats. Finally, oxA/hcrt-1-mediated excitatory synaptic transmission onto VTA neurons was not potentiated following an arousing, aversive stimulus, suggesting that oxA/hcrt-1-mediated glutamatergic synaptic transmission was potentiated selectively with highly salient positive reinforcers. These experiments provide evidence for a selective role of oxA/hcrt-1 signaling in motivation for highly salient reinforcers and may represent a unique opportunity to design novel therapies that selectively reduce excessive drive to consume positive reinforcers of high salience.

\section{Introduction}

The ventral tegmental area (VTA) is an important brain structure for relaying salient information (Berridge, 2007), and neural plasticity of dopamine neurons in this region plays an important role in early behavioral responses following initial drug exposures (Ungless et al., 2001; Borgland et al., 2004). It has been hypothesized that strengthened excitatory synapses onto dopamine neurons leads to burst-like firing, and consequently, enhanced dopamine release in VTA target regions involved in the attachment of salience to events associated with exposure to commonly addictive drugs (Jones and Bonci, 2005).

The orexins, also known as hypocretins, are neuropeptides produced in the lateral hypothalamic area that contribute to homeostatic processes such as arousal and feeding (de Lecea et al., 1998; Sakurai et al., 1998). They are comprised of two distinct peptides; orexin A/hypocretin-1 (oxA/hcrt-1) and orexin $\mathrm{B} /$ hypocretin-2. Orexin/hypocretin neurons project locally within the hypothalamus and widely throughout the brain, including a substantial projection to catecholaminergic regions such as the VTA and locus ceruleus (Peyron et al., 1998). Although orexin/

\footnotetext{
Received Dec. 19, 2008; revised July 28, 2009; accepted July 31, 2009.

This work was supported by a Natural Sciences and Engineering Research Council of Canada Discovery Grant (S.L.B.), National Alliance for Research on Schizophrenia and Depression Young Investigator Award (S.L.B.), National Institute on Drug Abuse Grant 1R01DA15096 (A.B.), and funding from the State of California for medical research on alcohol and substance abuse through the University of California, San Francisco (A.B.).

Correspondence should be addressed to Stephanie L. Borgland, Department of Anesthesiology, Pharmacology, and Therapeutics, The University of British Columbia, 212-2176 Health Sciences Mall, Vancouver, BC V6T 1Z3, Canada. E-mail: Borgland@interchange.ubc.ca.

D01:10.1523/JNEUROSC1.6096-08.2009

Copyright $\odot 2009$ Society for Neuroscience $\quad 0270-6474 / 09 / 2911215-11 \$ 15.00 / 0$
}

hypocretin neurons form only $5 \%$ of synapses in the VTA, the presence of intra-VTA orexin-containing dense core vesicles (Balcita-Pedicino and Sesack, 2007) indicates that these peptides are likely released extrasynaptically into the VTA to mediate its functional effects, including increasing firing rate (Korotkova et al., 2003; Muschamps et al., 2007), strengthening glutamatergic synapses (Borgland et al., 2006) and enhancing dopamine release in VTA target regions (Narita et al., 2006; Vittoz and Berridge, 2006; Vittoz et al., 2008).

Activation of orexin/hypocretin neurons contributes to homeostatic processes such as arousal and feeding (de Lecea et al., 1998; Sakurai et al., 1998). Selective activation of orexin/hypocretin neurons in mice using optogenetic technology promoted the transition from sleep to wakefulness (Adamantidis et al., 2007). Furthermore, orexin/hypocretin neuronal firing, identified by antidromic stimulation from the VTA, is linked to movement and EEG arousal during states that have strong emotional components, such as exploratory behavior and approaching food, suggesting a potential role for these neurons in arousal for goaldirected behaviors (Mileykovskiy et al., 2005). Accordingly, intracerebroventricular (i.c.v.) injections of oxA/hcrt-1 increases home cage food intake (Sakurai et al., 1998), and activation of orexin/hypocretin-1 receptors (ox/hcrt-1R) has been shown to play a role in palatable, high-fat food intake, as the ox/hcrt-1R antagonist, SB334867 reduced home cage feeding (Clegg et al., 2002; Zheng et al., 2007) and operant self-administration of highfat-containing food (Nair et al., 2008).

Recent evidence links the orexin system with reward and reinforcement (Aston-Jones et al., 2009). For example, orexin/ hypocretin neurons are activated when rats prefer contexts 
associated with addictive drugs or food (Harris et al., 2005). Further, reinstatement to cocaine (Harris et al., 2005; Boutrel et al., 2006; Smith et al., 2009) or ethanol (Lawrence et al., 2006), and the development of behavioral sensitization to cocaine (Borgland et al., 2006), is blocked by the ox/hcrt-1R antagonist, SB334867. We previously reported that oxA/hcrt-1 enhanced excitatory synaptic transmission in the VTA and also enabled plasticity associated with cocaine (Borgland et al., 2006), suggesting an underlying mechanism for the proposed reinforcing effects of oxA/hcrt-1. Because orexin/hypocretins are known to mediate both arousal and reward, we wanted to determine whether these peptides can drive motivated behavior. We hypothesized that by blocking ox/hcrt-1R signaling, we could reduce the effort rats are willing to exert for drug and palatable food reinforcers.

\section{Materials and Methods}

Individually housed male Sprague Dawley rats (350-400 g; Charles River Laboratories and the University of British Columbia) were used in all experiments. Animals were maintained on a $12 \mathrm{~h}$ light/dark cycle (lights on 7 A.M.-7 P.M.) with food and water available ad libitum unless stated below. Both behavioral experiments and decapitations for slice electrophysiology occurred at ZT $=8 \pm 1$ h (3 P.M.). All animal procedures were approved by the Institutional Animal Care and Use Committee of the Ernest Gallo Clinic and Research Center and the Animal Care Centre at the University of British Columbia.

Surgery. Animals were implanted with a chronically indwelling intravenous catheter and trained to self-administer cocaine, as previously described (Carelli et al., 2000). The catheter was flushed daily with heparinized saline $(0.4 \mathrm{ml}$ of $10 \mathrm{IU})$ to maintain patency. Some foodrestricted "sham" (FR) rats were fitted with intrajugular catheters. We found no difference in electrophysiological recordings of oxA/hcrt-1induced potentiation of NMDA receptor (NMDAR) currents between rats that had surgery and rats that did not have surgery, therefore we included rats from both groups together.

Cocaine self-administration. One week after surgery, animals were food-deprived for $24 \mathrm{~h}$ before 1 or 2 overnight shaping sessions. Rats were food restricted to $20 \mathrm{~g}$ of Purina rat chow per day delivered in the home cage for the duration of the experiment. Shaping for lever pressing occurred during overnight $(15 \mathrm{~h})$ and fixed ratio 1 (FR1) sessions in standard operant chambers with two retractable levers, each beneath a cue light and flanking a food hopper (Coulbourn Instruments). Animals were randomly assigned an active lever for the duration of experimentation. During shaping, each active lever press resulted in the delivery of a single $45 \mathrm{mg}$ Noyes food pellet (Research Diets), in addition to a $4 \mathrm{~s}$ cue light illumination over the active lever. Each pellet delivery was followed by an $18 \mathrm{~s}$ timeout where additional lever presses were recorded, but did not result in food reinforcement or presentation of the cue light. The day following 1 or $215 \mathrm{~h}$ overnight sessions, animals were placed in a $2 \mathrm{~h}$ session where food was delivered on an FR1 schedule, and every eighth press resulted in a $0.50 \mathrm{mg} / \mathrm{kg}$ cocaine infusion over $2 \mathrm{~s}$, which was paired with both $4 \mathrm{~s}$ cue light illumination and a tone. Each infusion was followed by an $18 \mathrm{~s}$ timeout. The animals were limited to earning 200 reinforcers during all sessions. The session with both food and cocaine as reinforcers facilitated the transition to cocaine-only self-administration. After this food-cocaine training session, animals received 14-17 additional days of training. Each day, animals were allowed to self-administer cocaine on an FR1, then FR3, and finally an FR5 schedule, with each infusion paired with a $4 \mathrm{~s}$ cue light and a tone followed by an $18 \mathrm{~s}$ timeout period. Once rats achieved adequate lever pressing on an FR5 schedule (rats received a minimum of 9 infusions on each FR5 training session), rats were tested daily under a progressive ratio (PR) schedule of reinforcement for $4 \mathrm{~d}$. Under this schedule, the number of responses required to obtain each successive infusion of cocaine was determined by the equation (Richardson and Roberts, 1996): Response ratio $=\left[5 e^{(\text {injection number } \times 0.2)}\right]-5$, to produce the following sequence of required lever presses: 1, 2, 4, 6, 9, 12, 15, 25, 32, 40, 50, 62, etc. The daily PR sessions were terminated when $1 \mathrm{~h}$ elapsed without a drug infusion and, as a result, generally lasted $3 \mathrm{~h}$ or less. The maximal number of presses emitted to attain the final ratio was defined as the breakpoint (Richardson and Roberts, 1996). There was no effect of time on lever pressing, reinforcers received, or breakpoint over the 4 days of PR (supplemental Fig. 1, available at www.jneurosci.org as supplemental material). During all sessions, depression of the inactive lever generated no programmed consequence, but responses were recorded.

Regular food and high fat chocolate pellet self-administration. The training protocol for food self-administration was similar to that described above for cocaine self-administration, with 2 overnight shaping sessions ( $15 \mathrm{~h}, \mathrm{FR} 1$ ) where pressing on the active lever delivered one $45 \mathrm{mg}$ pellet as well as cue light illumination and a tone. Each reinforcer was followed by an $18 \mathrm{~s}$ timeout. Animals were mildly food restricted, as above, for the entire set of operant sessions. Because rats appeared to respond more frequently for food, the PR schedule was calculated by the following equation (Richardson and Roberts, 1996): Response ratio $=\left[5 e^{\text {(injection number } \times 0.24)}\right]-5$, to produce the following sequence of required lever presses: $1,3,5,8,12,16,22,29,38,50,65,84$, etc. Separate groups of rats were trained to lever press for either standard food, or high fat chocolate pellets ( $45 \mathrm{mg}$ pellet containing $35 \%$ fat and $37 \%$ carbohydrate with chocolate flavor and scent, Bioserv). These experiments were conducted in a manner similar to that described above.

On the second and third days of PR testing, rats that were trained to lever press for either cocaine, regular food (RF) pellets, or high fat chocolate (HF) pellets received vehicle injections (i.p) and on the subsequent day received an injection of the ox/hcrt-1R antagonist, SB334867 (10 or $20 \mathrm{mg} / \mathrm{kg})$. In some experiments, cocaine $(15 \mathrm{mg} / \mathrm{kg})$, the dopamine uptake blocker GBR12909 (5 mg/kg), or saline was given on the second, third and fourth days of PR testing.

Effort-based task. Rats were trained and tested in a 4-arm black acrylic cross-maze (dimensions: $60 \mathrm{~cm}$ long, $10 \mathrm{~cm}$ wide, $10 \mathrm{~cm}$ deep) with rectangular food wells ( $4 \mathrm{~cm}$ wide $\times 1 \mathrm{~cm}$ deep) placed $2 \mathrm{~cm}$ from the end wall of each arm. A removable piece of black acrylic blocked one of the arms of the maze to for a " $\mathrm{T}$ " configuration throughout the duration of the experiment, including training. Rats were released from the stem arm located in the same spatial location in the testing room. One or both choice arms of the maze could be blocked with barriers over which the animals had to climb to obtain rewards. These barriers were constructed in the shape of a wedge, using galvanized wire mesh with white plastic sides $30 \mathrm{~cm}$ in length. To obtain access to the food in one arm, rats had to scale up the vertical side and descend down a slope of varying incline depending on the size of the barrier. Habituation and training were identical to procedures described by Floresco and Ghods-Sharifi (2007) and outlined in the time line in Figure 4A. Briefly, in the first phase of discrimination training, 3 pellets of high fat chocolate food were put in the feeding well of the goal arm (the HF arm) and 3 regular food pellets were put in the other goal arm (the RF arm). For half of the rats, the HF arm was the left arm and for the others, it was the right. Each rat was placed in the stem arm and was allowed to sample from either the HF or RF arm. For the first $3 \mathrm{~d}$, each rat could sample from both arms for 5 trials. On the next $3 \mathrm{~d}$, rats were moved to the second phase of discrimination training in which access to one of the goal arms was prevented by blocking the arms with a plastic block at its entrance, forcing the rat to sample a particular arm on each trial. The order of these forced trials was determined pseudorandomly so that they never had more than two consecutive turns in either direction for a total of 10 trials per day. In the following discrimination training, rats had free choice to either the HF or the RF arm for 16 trials. Rats were removed from the maze after eating the food in the selected arm without being able to sample the other arm. On trials 6 and 12, the plastic block was placed in the arm chosen by the rat on the previous trial to prevent potential turn biases from interfering with discrimination training. For discrimination and subsequent barrier training, rats were trained to reach a criterion performance of eating from the HF arm on $\geq 75 \%$ of trials in a daily session. When a rat achieved criterion performance for 2 consecutive days, it was taken to the next phase of the task where the wire mesh barriers were introduced.

For the first $2 \mathrm{~d}$ of barrier training rats received a total of 16 trials with the small ( $15 \mathrm{~cm}$ barrier) placed in the HF arm. On the first 8 trials, rats were forced to scale the barrier to receive 3 high fat pellets. On the sub- 
sequent 8 trials, rats were allowed to sample from both arms and were removed from the maze once rats made their first choice. On all subsequent trials, rats received a total of 18 trials per day, the first two of which were forced choice; for the subsequent 16 trials, rats were free to choose either the HF or RF arm. Rats were removed from the maze once they chose an arm. Each rat received daily training sessions until it achieved a criterion performance for 2 consecutive days. Fifteen minutes before the next session, rats were injected with vehicle solution (10\% DMSO, $10 \%$ $\beta$-hydroxypropylcyclodextrin in distilled water, i.p.), and the following day rats were injected with SB334867 (10 mg/kg, i.p.) 15 min before the session.

After testing, the barrier size was increased to $30 \mathrm{~cm}$, and training continued until the rat again achieved criterion performance for 2 consecutive days. The day after achieving criterion performance with the 30 $\mathrm{cm}$ barrier in the HF arm for 2 consecutive days, rats received a vehicle injection and were then tested on the $30 \mathrm{~cm}$ barrier 15 min later. The following day, rats received an injection of SB334867 (10 mg/kg) $15 \mathrm{~min}$ before being tested on the $30 \mathrm{~cm}$ barrier.

Pavlovian approach conditioning. Twenty-two male Sprague Dawley rats were restricted to $85 \%$ body weight for 1 week before conditioning. At the same time each day, animals were given an injection of either SB-334867 (15 mg/kg, i.p.) or vehicle (10\% DMSO and $2 \%$ $\beta$-cyclodextrin in water) 15 min before conditioning. Animals were then placed in operant chambers (Med Associates) for a conditioning session that lasted $\sim 30 \mathrm{~min}$. The conditioning sessions were similar to that described by Stuber et al. (2008). Presentation of a $10 \mathrm{~s}$ conditioned stimulus (CS; a $5 \mathrm{kHz}$ tone and illumination of two stimulus lights) occurred on a variable interval $30 \mathrm{~s}$ schedule. The termination of the CS was followed by delivery of two $45 \mathrm{mg}$ food pellets (Bioserv) into a food cup located in an alcove between the two stimulus lights. The alcove contained an infrared photobeam that detected approaches toward the food cup. During each of seven conditioning sessions, rats received 30 presentations of the CS and food. Over these sessions, approaches toward the food cup during the intertrial interval delayed the onset of the CS by $3 \mathrm{~s}$. The primary dependent measure of interest was the proportion of nosepokes directed toward the food cup during presentation of the CS relative to the number of total nosepokes made during the session (a measure of discriminative pavlovian approach).

Stress manipulations. Similar to self-administration experiments, rats were restricted to $20 \mathrm{~g}$ of food per day for the duration of the experiment. Each day for 7 consecutive days, rats experienced unpredicted, intermittent footshocks in 30 min sessions delivered in operant chambers identical to those described above except that levers remained retracted throughout experimentation. A house light and exhaust fan operated during each session. Inescapable and unsignaled footshocks $(0.8 \mathrm{~mA}$, $0.1 \mathrm{~s}$ duration) were delivered on a variable interval $40 \mathrm{~s}$ schedule across a series of 8 floor poles at $40 \mathrm{~Hz}$. Rats received a maximum of 35 shocks per session. After the session, rats were returned to the home cage and fed.

Stress hormone measurement. Blood $(\sim 600 \mu \mathrm{l})$ was collected from the left ventricle of pentobarbital-anesthetized rats just before aCSF perfusion and decapitation for midbrain slicing. Blood was immediately mixed with $60 \mu \mathrm{l}$ of cold $48.3 \mathrm{~mm}$ EDTA. Samples were then centrifuged at $2000 \mathrm{RCF}$ for $20 \mathrm{~min}$ at $4^{\circ} \mathrm{C}$, serum decanted, and stored at $-80^{\circ} \mathrm{C}$ until use. ${ }^{125} \mathrm{I}$ labeled radioimmunoassays for ACTH (MP Biosciences) were performed according to manufacturer's instructions in duplicate or triplicate with the resulting supernatant decanted and pellet counted in a gamma counter (Beckmann Coulter) according to assay requirements. Nonspecific binding for ACTH was 7.9\%. The percentage bound standard curve $r^{2}$ was 0.97 using a logarithmic fit. Serum concentrations were determined from this curve.

OxA/Hcrt-1 peptide measurement. Procedures for detection of oxA/ hcrt-1 expression were performed in triplicate as described previously (Porkka-Heiskanen et al., 2004) and according to manufacturer instructions (Peninsula Laboratories). The region of lateral hypothalamus approximating that sampled for electrophysiological experiments was excised from a series of $250 \mu \mathrm{m}$ horizontal slices in cold perfusion buffer using a scalpel for a total sample size of $2 \times 2.5 \mathrm{~mm}$ per hemisphere. Samples were immediately stored at $-80^{\circ} \mathrm{C}$ until whole cell lysates were obtained via homogenization on ice in cold $1 \%$ trifluoroacetic acid using
70 strokes of a glass/Teflon homogenizer. Samples were centrifuged at $12,000 \times g$ for $20 \mathrm{~min}$ at $4^{\circ} \mathrm{C}$, supernatant lyophilized, and resuspended in $0.5 \mathrm{ml}$ of the provided buffer diluted with Millipore water. Samples $(100 \mu \mathrm{l})$ were processed in triplicate and total protein content determined by the method of Lowry et al. (1951) from a $10 \mu$ laliquot. Results are expressed as pg oxA/hcrt-1 per mg protein. The intra-assay coefficient of variation was $3.29 \%$.

Electrophysiology. All the electrophysiological recordings were performed in adult rats ranging from 350 to $400 \mathrm{~g}$. Briefly, rats were deeply anesthetized with pentobarbital and perfused with ice-cold artificial CSF (aCSF) including (in mM): 225 sucrose, $119 \mathrm{NaCl}, 2.5 \mathrm{KCl}, 1.0 \mathrm{NaH}_{2} \mathrm{PO}_{4}$, $4.9 \mathrm{MgCl}_{2}, 0.1 \mathrm{CaCl}_{2}, 26.2 \mathrm{NaHCO}_{3}, 1.25$ glucose, 3 kynuretic acid. The brain was extracted and horizontal sections of the VTA $(230 \mu \mathrm{M})$ were prepared with a Vibratome (Leica). Slices were placed in a holding chamber and allowed to recover for at least $1 \mathrm{~h}$ before being placed in the recording chamber and superfused with bicarbonate-buffered solution (aCSF) saturated with 95\% $\mathrm{O}_{2} / 5 \% \mathrm{CO}_{2}$ and containing (in $\mathrm{mm}$ ): 119 $\mathrm{NaCl}, 1.6 \mathrm{KCl}, 1.0 \mathrm{NaH}_{2} \mathrm{PO}_{4}, 1.3 \mathrm{MgCl}_{2}, 2.5 \mathrm{CaCl}_{2}, 26.2 \mathrm{NaHCO}_{3}$ and 11 glucose (at $\left.32-34^{\circ} \mathrm{C}\right)$. Picrotoxin $(100 \mu \mathrm{M})$ was added to block $\mathrm{GABA}_{\mathrm{A}}$ receptor-mediated IPSCs. Cells were visualized using infrared differential interference contrast video microscopy. Whole-cell voltage clamp recordings were made using an Axopatch 1D amplifier (Molecular Devices). Electrodes (2.8-4.0 M $\Omega$ ) contained (in $\mathrm{mM}$ ): 120 cesium methanesulfonate, 20 HEPES, 0.4 EGTA, $2.8 \mathrm{NaCl}, 5$ TEA-Cl, 2.5 MgATP, and $0.25 \mathrm{NaGTP}, \mathrm{pH}$ 7.2-7.3 (270-285 mOsm). Series resistance (10-30 $\mathrm{M} \Omega$ ) and input resistance were monitored online with a $4 \mathrm{mV}$ depolarizing step (50 ms) given just after every afferent stimulus. Dopaminergic VTA neurons were identified by the presence of a large $I_{\mathrm{h}}$ current (Lacey et al., 1990; Johnson and North, 1992). A bipolar stimulating electrode was placed $100-300 \mu \mathrm{m}$ rostral to the recording electrode and was used to stimulate excitatory afferents at $0.1 \mathrm{~Hz}$. Neurons were voltageclamped at $+40 \mathrm{mV}$ to record NMDAR-mediated EPSCs. EPSCs were filtered at $2 \mathrm{kHz}$, digitized at $5-10 \mathrm{kHz}$ and collected online using Igor Pro software (Wavemetrics). NMDAR traces were constructed by averaging 12 EPSCs ( $120 \mathrm{~s}$ ) elicited at $+40 \mathrm{mV}$. NMDAR EPSCs were evoked at $+40 \mathrm{mV}$ and measured $20 \mathrm{~ms}$ after the stimulus artifact, a time point whereby AMPAR currents have decayed. Miniature EPSCs (mEPSCs) were recorded in neurons voltage-clamped at $-70 \mathrm{mV}$ in the presence of tetrodotoxin $(500 \mathrm{nM})$ to block action potential-driven spontaneous events. A within-cell design was used whereby mEPSCs were recorded before and $10 \mathrm{~min}$ after a $5 \mathrm{~min}$ application of oxA/hcrt-1 (100 nM). mEPSCs were selected based on their amplitude $(>8 \mathrm{pA})$, decay time $(<3 \mathrm{~ms})$, and rise time $(<1 \mathrm{~ms})$ using the MiniAnalysis program (Synaptosoft).

Drugs. SB334867 (10 mg/kg or $20 \mathrm{mg} / \mathrm{kg})$ was dissolved in a vehicle solution containing $25 \% \beta$-cyclodextrin, $5 \%$ DMSO and $0.9 \%$ saline, and administered in a volume of $3 \mathrm{ml} / \mathrm{kg}$. GBR12909 and cocaine were dissolved in $0.9 \%$ saline, and administered in a volume of $3 \mathrm{ml} / \mathrm{kg}$.

Data analysis. All values are expressed as mean \pm SEM. Statistical significance was assessed using a Repeated Measures ANOVA for multiple group comparisons of the same rats over 4 test days. A Bonferroni post hoc test following an ANOVA was used to test significant differences between groups. For electrophysiological experiments, a one-way ANOVA was used for multiple group comparisons with a Bonferroni post hoc test, unless otherwise indicated.

\section{Results}

\section{Blockade of ox/hcrt-1R reduced the breakpoint for highly salient reinforcers}

To establish whether the work required to obtain cocaine $(0.5$ $\mathrm{mg} /$ infusion), regular food (RF) pellets (fat: $5 \%$; carbohydrate: $72 \%$ ) or high fat chocolate-flavored (HF) food pellets (fat: $35 \%$; carbohydrate: $37 \%$ ) was altered by blockade of ox/hcrt-1R signaling, rats were tested under a progressive ratio (PR) schedule whereby the response requirement to earn a drug infusion or food pellet escalated after the delivery of each reinforcer. The maximal number of responses emitted to successfully complete the final ratio was defined as the breakpoint and is hypothesized to reflect 
the animal's motivational limit (Richardson and Roberts, 1996). SB334867 (10 $\mathrm{mg} / \mathrm{kg}$, i.p.) significantly reduced the breakpoint in cocaine self-administering rats (Fig. $1 A$ ) compared with their performance after vehicle injection. The final ratio achieved in the presence of SB334867 was $20 \pm 5$ compared with $37 \pm 9$ in vehicle treatment $\left(p<0.05, F_{(3,33)}=4.370\right.$, $n=12$ ) (Fig. 1A). Significant divergence of cumulative presses for cocaine after SB334867 treatment compared with vehicle rats occurred when the response requirement for reinforcement increased (Fig. $1 B ; p<0.05$ at $160 \mathrm{~min}$ ). In contrast, the breakpoint was not changed in rats lever pressing for RF after injection of SB334867 at either $10 \mathrm{mg} / \mathrm{kg}(n=10)$ or $20 \mathrm{mg} / \mathrm{kg},(n=12)$ doses $\left(F_{(3,44)}=\right.$ 0.25) (Fig. $1 A, C$ ). Cumulative presses for $\mathrm{RF}$ after SB334867 treatment $(20 \mathrm{mg} / \mathrm{kg})$ was not different from that observed after vehicle treatment $(p>0.05$ at $160 \mathrm{~min}$, $n=12$, Fig. $1 C$ ). However, breakpoint for HF pellets was significantly reduced by SB334867 pretreatment at the lower dose $(10 \mathrm{mg} / \mathrm{kg})$ and was ineffective at reducing work exerted for RF (32 \pm 9$)$ compared with vehicle-treated rats $(69 \pm 11$, $p<0.05 ; F=11.52 ; n=12$ ) (Fig. $1 A$ ). Furthermore, when the work-effort requirement became greater, cumulative presses for HF pellets were significantly less after SB334867 relative to vehicle $(p<0.05$ at $20 \mathrm{~min}$ ) (Fig. $1 D$ ). Together, these results suggest that ox/hcrt-1R signaling may selectively modulate the amount of work that a rat is willing to produce to obtain cocaine or HF pellets, but not a less salient reward such as RF.

To determine whether the reduction of work required to obtain HF pellets was due to the ox/hcrt-1R antagonist mediating satiety, we measured the amount of freely obtained RF or HF pellets consumed after $15 \mathrm{~min}$. There was no significant difference in the amount of RF $(n=8)$ or $\operatorname{HF}(n=9)$ pellets eaten after either vehicle (RF: $5.9 \pm 1.9$, HF: $6.8 \pm 0.8$ ) or SB334867 (RF: $5.8 \pm 1.7$, HF: $6.6 \pm 0.9,10 \mathrm{mg} / \mathrm{kg}$, i.p. $)$ injection $(p>0.05)$. These data suggest that the dose of SB334867, which significantly reduced work exerted to obtain cocaine or HF, did not affect satiety in our paradigm.

Elevation of extracellular dopamine levels in the nucleus accumbens has been hypothesized to enhance the reinforcing properties of other paired stimuli. For example, after amphetamine administration, which increases dopamine levels, rats exhibit an enhanced motivation for RF under a PR schedule of reinforcement (Zhang et al., 2003). To assess how ox/hcrt-1R signaling may modulate the ability of increased dopamine transmission to amplify motivated responding, we gave systemic injections of cocaine $(15 \mathrm{mg} / \mathrm{kg}$, i.p.) or the selective dopamine transporter blocker, GBR 12909 (GBR; 5 mg/kg, i.p.) on days 2-4 of PR testing in a separate group of rats trained to lever press for RF pellets. Cocaine (Fig. 2A) or GBR (Fig. $2 B$ ) administration significantly increased the breakpoint compared with responding before treatment $\left(F_{(3,38)}=5.08, p<0.05\right)$. SB334867 $(10 \mathrm{mg} / \mathrm{kg})$ in the presence of cocaine or GBR blocked the increased breakpoint (Fig. $2 A, B$ ). The final ratio obtained in the presence of
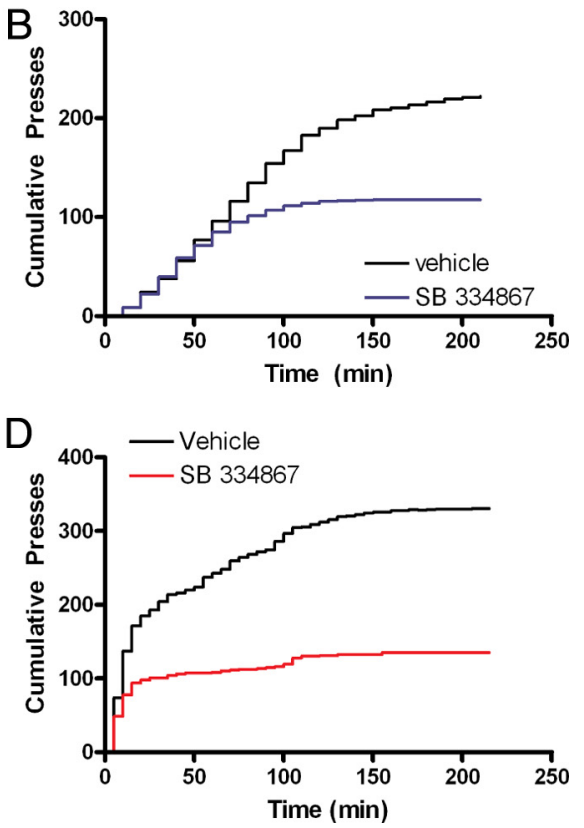

Figure 1. $0 x /$ hcrt-1R signaling is involved in work for highly salient reinforcers. $A, S B 334867(10 \mathrm{mg} / \mathrm{kg})$ significantly reduced were significantly reduced after SB334867 (10 mg/kg; red line) compared with cumulative presses after a vehicle injection (black line) on the previous day ( $n=12, p<0.05$ at $20 \mathrm{~min}$ ). Bars represent mean \pm SEM. ${ }^{* *} p<0.001$.

SB334867 and cocaine $(78 \pm 12, n=12)$ or SB334867 and GBR $(142 \pm 22, n=11)$ was significantly reduced compared with rats treated with either cocaine $(165 \pm 31, n=12, p<0.05)$ or GBR (332 $\pm 57, n=11, p<0.05)$ alone. Finally, there was no significant difference in breakpoint for RF when rats were given cocaine with SB334867 compared with no treatment or vehicle treatment $(p>0.05)$ (Fig. $2 C$ ). These results suggest that while $\mathrm{ox} /$ hcrt-1R signaling does not appear to modulate the motivation to work for RF, ox/hcrt-1R-mediated signaling is nonetheless involved in modulating the motivation to work for RF when the apparent motivational quality has been increased by substances known to elevate extracellular dopamine concentrations.

\section{Blockade of ox/hcrt-1R reduced effort-related choice of high fat chocolate food over regular food}

To further explore the nature of how ox/hcrt-1R-mediated signaling modulates motivated behavior, we tested whether SB334867 modulated the rats' willingness to expend more effort to obtain HF over RF pellets (Fig. $3 A-C$ ). Rats were trained to criterion performance ( $\geq 75 \%$ high fat chocolate food arm entries for 2 consecutive days) on a "T-maze" with a mesh grid barrier at the entrance of the HF arm and free access to the regular food arm. The rats required $24 \pm 4$ training days to meet criterion performance on the $15 \mathrm{~cm}$ barrier and $29 \pm 5$ training days to meet criterion performance on the $30 \mathrm{~cm}$ barrier. Three of 12 animals did not meet criterion on the $15 \mathrm{~cm}$ barrier and were removed from the experiment. Of the remaining 9 animals, only 8 made it to criterion for the $30 \mathrm{~cm}$ barrier. Following injection of SB334867 (10 mg/kg, i.p), rats significantly reduced HF arm entries $(71 \pm 6 \%)$ compared with vehicle treated $(92 \pm 1 \%$, $p<$ $0.05)$ or naive $(86 \pm 3 \%, p<0.01)$ rats trained on the $15 \mathrm{~cm}$ 

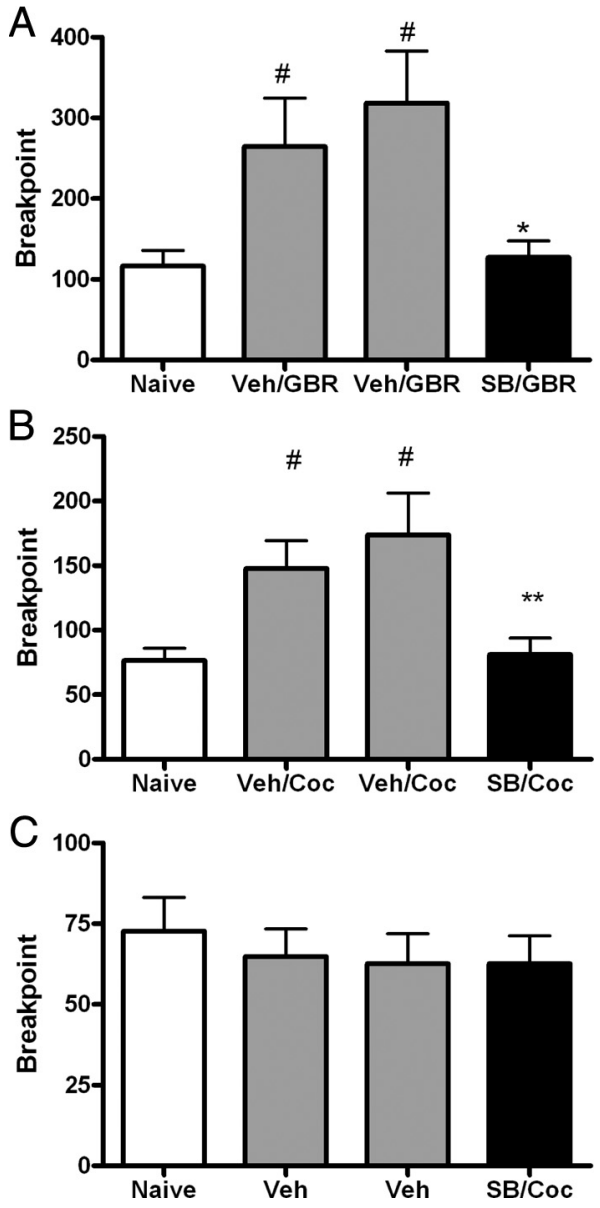

Figure 2. SB334867 blocks cocaine-potentiated food seeking. Rats learned to lever press for cocaine $(0.5 \mathrm{mg} /$ infusion), regular, or high fat chocolate food on a fixed ratio schedule. Each reinforced lever press on the active lever was associated with a tone and a light cue. Once stability criteria on Fixed Ratio 5 were met, rats were switched to a progressive ratio schedule of reinforcement for 4 consecutive days. Rats received i.p. injections of vehicle (Veh) and/or cocaine (Coc) (15 mg/kg) or GBR 12909 (GBR) (5 mg/kg) on days 2 and 3 and of the ox/hcrt-1R antagonist, SB334867 (SB) on day 4. A, Cocaine injections (15 mg/kg, i.p.) on days 2 and 3 significantly increased the breakpoint for regular food ( $\left.{ }^{\#} p<0.05\right)$. SB334867 $(10 \mathrm{mg} / \mathrm{kg})$ injected $15 \mathrm{~min}$ before cocaine $(15 \mathrm{mg} / \mathrm{kg}$ ) significantly reduced the breakpoint for regular food (shaded bar) compared with that after vehicle injections $\left(n=11,{ }^{* *} p<0.01\right)$. B, GBR injections $(5 \mathrm{mg} / \mathrm{kg}$, i.p.) on days 2 and 3 significantly increased the breakpoint for regular food $\left({ }^{\#} p<0.05\right)$. SB334867 (10 mg/kg) injected $15 \mathrm{~min}$ before GBR $(5 \mathrm{mg} / \mathrm{kg})$ significantly reduced the breakpoint for regular food (black bar) compared with that after vehicle injections ( $n=12$, $\left.{ }^{*} p<0.05\right)$. C, Rats received a vehicle injection (i.p.) on days 2 and 3 and an injection of SB334867 (10 mg/kg, i.p) with cocaine ( $15 \mathrm{mg} / \mathrm{kg}$, i.p.) on day 4. In the presence of SB334867, a single cocaine exposure did not potentiate the breakpoint in regular food self-administering rats $(p>0.05)$. Bars represent mean and SEM of 11 rats. Repeated measures ANOVA was used.

barrier $\left(n=9 ; F_{(2,26)}=11.1\right)$ (Fig. $\left.3 B\right)$. On the $30 \mathrm{~cm}$ barrier, SB334867 also significantly reduced HF arm entries (58 $\pm 2 \%$ ) compared with vehicle treatment $(92 \pm 2 \%, p<0.001)$ or no treatment $\left(89 \pm 2 \%, p<0.001, n=8 ; F_{(2,23)}=72.5\right)$ (Fig. $3 C$ ). Regardless of the baseline performance, each rat made substantially fewer entries into the barriered HF arm following injection of SB334867. Thus, under normal conditions, rats will work to obtain HF over RF. However, this preference is potently reduced by administration of an ox/hcrt-1R antagonist.

\section{Ox/hcrt-1R blockade does not modulate conditioned pavlovian approach}

$\mathrm{Ox} / \mathrm{hcrt}-1 \mathrm{R}$ signaling has been reported to underlie cue-associated reinstatement of ethanol (Lawrence et al., 2006) or cocaine- seeking (Smith et al., 2009). Perhaps ox/hcrt-1R signaling underlies the learning of cues that may be guiding the rats to obtain relevant reinforcers. To assess this possibility, we tested how blockade of ox/hcrt-1 signaling may affect the acquisition of discriminative pavlovian approach induced by stimuli paired with sucrose delivery. Rats underwent 7 daily conditioning sessions in which the onset of a cue light and tone preceded the delivery of two sucrose pellets (Fig. 3D). Cue-reward learning was assayed by the development of conditioned approach behavior in which rats displayed an increase in the proportion of goal-directed nosepokes into the pellet receptacle during presentation of the CS relative to nosepokes made during the intertrial interval (i.e., the discrimination ratio). Before each of the 7 training sessions, rats received injections of either vehicle $(n=11)$ or SB334867 $(n=$ 11 ). As shown in Figure $3 E$, rats in both groups showed an increase in the proportion of nosepokes during the CS over $7 \mathrm{~d}$ of training. Analysis of these data revealed a significant main effect of training day $\left(F_{(6,132)}=26.11, p<0.01\right)$. However, there was no significant main effect of treatment group or treatment by training day interaction (both $F s<1$, n.s.). These data indicate that blockade of ox/hrct-1R signaling does not disrupt simple pavlovian approach learning. Thus, ox/hrct-1R activation may contribute to a bias toward high cost/high palatable food options, but does not appear to be required for learning an approach response induced by conditioned cues.

\section{OxA/hcrt-1-induced plasticity in the VTA is enhanced following cocaine or HF self-administration}

To determine whether cocaine, RF or HF pellet seeking enhanced oxA/hcrt-1-mediated glutamatergic synaptic transmission in the VTA, NMDAR evoked EPSCs (eEPSCs) were recorded using patch-clamp electrophysiology. To examine oxA/hcrt-1-mediated plasticity induced by operant history without the confounding aspect of a more complex $\mathrm{PR}$ responding for cocaine, RF, or HF seeking, the paradigm was modified so that rats received reinforcement on a modified, fixed schedule (fixed ratio 1 followed by a $20 \mathrm{~s}$ timeout). Recordings of VTA neurons from midbrain slices were made $20-24 \mathrm{~h}$ after rats obtained stable selfadministration of cocaine, RF, or HF pellets (typically 14-17 d).

OxA/hcrt-1 (100 nM, $5 \mathrm{~min}$ ) significantly potentiated NMDARmediated eEPSCs in naive adult rats (Fig. $4 A, B$ ). Food restriction (FR; $20 \mathrm{~g}$ of food per day as above) did not significantly alter oxA/hcrt-1-mediated plasticity in the VTA compared with naive rats (maximum potentiation in naive: $123 \pm 7 \%, n=7$ vs sham: $129 \pm 3 \%, n=7, p>0.05$ ) (Fig. $4 A-C$ ). Similarly, RF pellet self-administration also did not alter oxA/hcrt-1-mediated potentiation of NMDAR currents $(124 \pm 10 \%, n=7, p>0.05)$ (Fig. $4 A-C$ ). In contrast, a significantly elevated oxA/hcrt-1mediated potentiation of NMDAR eEPSCs (cocaine: $159 \pm 10 \%$, $n=9$; HF: $156 \pm 12 \%, n=9$ ) (Fig. $4 A-C$ ) was observed in cocaine or HF pellet self-administering rats compared with naive, sham or RF self-administering rats $(p<0.05)$.

The lateral hypothalamus was removed from horizontal VTA slices used for electrophysiology to determine whether prior selfadministration history altered the expression of oxA/hcrt-1. Although there was a significant increase in oxA/hcrt-1 protein of $\mathrm{RF}$ and HF self-administering rats compared with naive rats $(p<$ $0.05)$, no significant differences in oxA/hcrt-1 protein levels were found between naive and food-restricted rats, nor between RF, cocaine, or HF self-administration (Fig. $4 D)(p>0.05$, one way ANOVA, Dunnett's multiple comparison test). 
Potentiation of orexin A-mediated glutamatergic synaptic transmission is presynaptic

To determine the locus of synaptic change, we recorded the frequency and amplitude of AMPAR mEPSCs before (baseline) and $10 \mathrm{~min}$ after a $5 \mathrm{~min}$ bath application of oxA/hcrt-1 (100 nM). Frequency of mEPSCs was significantly increased by oxA/hcrt-1 (100 nM) in cocaine (before: $1.0 \pm 0.2 \mathrm{~Hz}$ vs after: $1.3 \pm 0.1$, $n=8, p<0.05$, paired $t$ test) (Fig. $5 B, E_{i}$ ) and HF (before: $1.3 \pm 0.3 \mathrm{~Hz}$ vs after: $1.6 \pm 0.4, n=7, p<0.05$, paired $t$ test) (Fig. $5 C, F_{i}$ ) self-administering rats compared with RF self-administering rats (before: $0.9 \pm 0.1 \mathrm{~Hz}$ vs after: $0.8 \pm 0.1, n=8$, $p>0.05$, paired $t$ test) (Fig. $5 A, D_{i}$ ). OxA/ hcrt-1 (100 nM) did not alter mEPSC amplitude in either RF (before: $11.3 \pm 0.5 \mathrm{pA}$ vs after: $11.0 \pm 0.8 \mathrm{pA}, n=8, p>0.05)$ (Fig. $5 A, D_{i i}$ ), cocaine (before: $12.4 \pm 0.6$ pA vs after: $12.3 \pm 0.8 \mathrm{pA}, n=8, p>0.05)$ (Fig. $5 B, E_{i i}$ ) or HF (before: $10.7 \pm 0.5 \mathrm{pA}$ vs after: $9.8 \pm 0.4 \mathrm{pA}, n=7, p>0.05)$ (Fig. $5 C, F_{i i}$ ) self-administering rats. Because oxA/hcrt-1 increased frequency, but not amplitude of mEPSCs in cocaine or HF selfadministering rats, these results suggest that a presynaptic increase in glutamatergic transmission occurs following cocaine or HF self-administration.

To test whether prior self-administration resulted in a modification of ox/ hcrt-1R sensitivity, a concentration of oxA/hcrt-1 (1 nM) that did not potentiate eEPSCs in VTA neurons of naive rats was tested on NMDAR eEPSCs in VTA neurons of RF, cocaine and HF selfadministering rats (Fig. 6A) $(n=7)$. OxA/hcrt-1 significantly potentiated NMDAR currents in VTA neurons of cocaine $(123 \pm 4 \%, n=7, p<0.05)$ and HF self-administering rats $(123 \pm 6 \%$, $n=8, p<0.05)$, but not RF selfadministering rats $(104 \pm 3 \%, n=8$, $p>0.05)$. These results suggest an alteration in the efficacy or availability of ox/hcrt-1Rs and/or cognate signal transduction machinery on excitatory VTA inputs to the VTA after cocaine or HF self-administration, but not RF self-administration.

Enhanced oxA/hcrt-1-mediated glutamatergic synaptic transmission in the VTA is important for salient positive reinforcers, but not aversive arousing stimuli

Potentiated excitatory synaptic transmission observed after cocaine or HF self-administration may be due to the arousing properties of these reinforcers as opposed to their incentive properties. Thus, we sought to determine whether inducing arousal with an aversive stimulus could potentiate oxA/hcrt-1-mediated excitatory synaptic transmission in the VTA in a manner similar to

$\mathrm{D}$
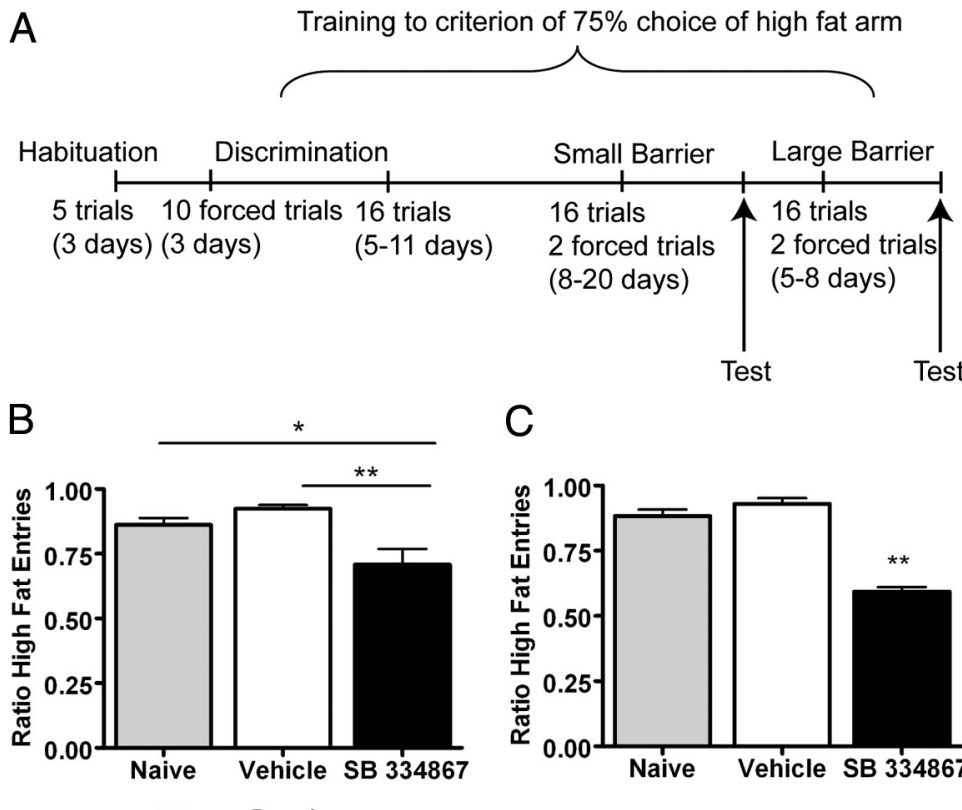

C
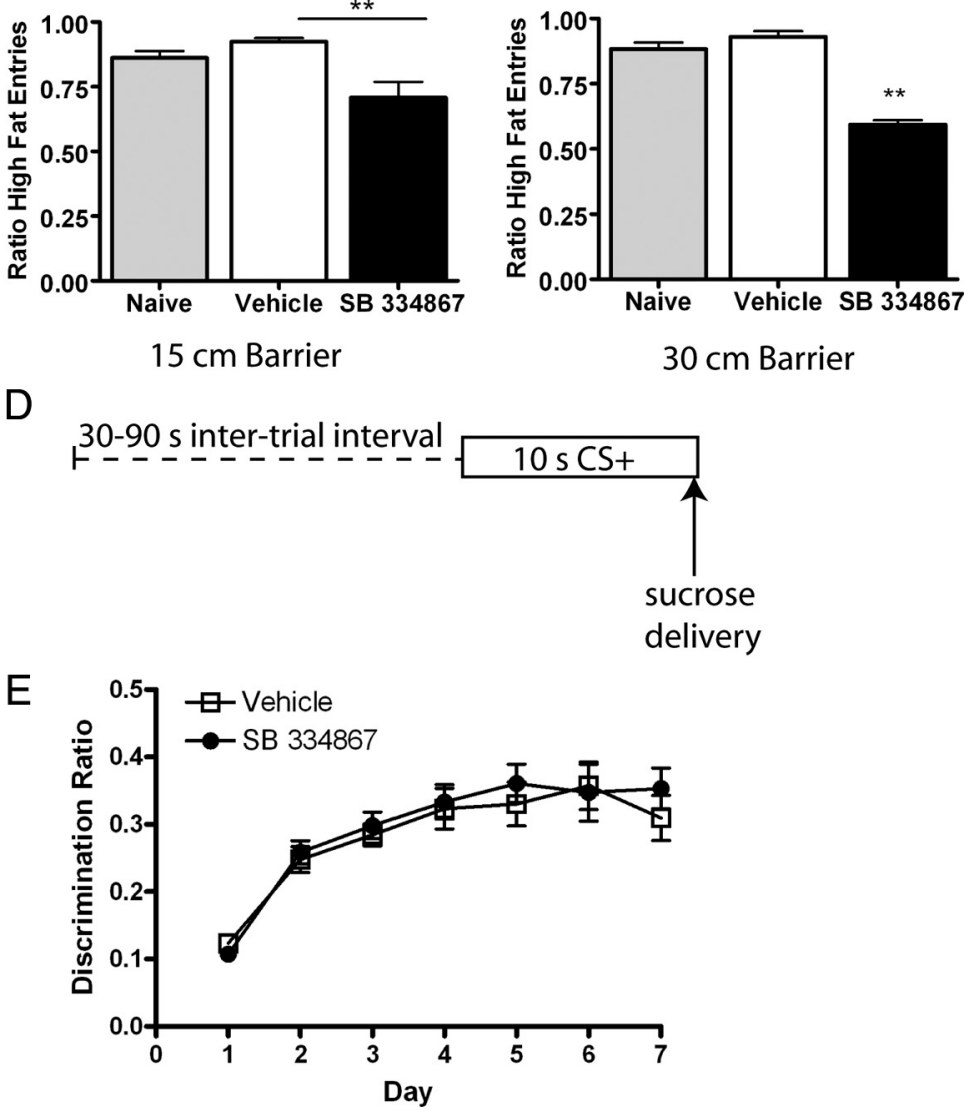

Figure 3. SB334867 reduces the effort, but not cue-learning for palatable reinforcers. In the effort based task, rats had free access to one arm on the T-maze baited with 3-45 mg of pellets of regular food or could climb a barrier to obtain equal pellets of high fat chocolate food. $\boldsymbol{A}$, Timeline depicting the habituation, training and testing procedures used. $\boldsymbol{B}, \boldsymbol{C}$, Rats must $\operatorname{limb}(\boldsymbol{B})$ a 15 $\mathrm{cm}$ barrier ( $n=8$ rats) or ( $C$ ) $30 \mathrm{~cm}$ barrier ( $n=9$ rats) to obtain high fat food. Bars represent mean and SEM of the ratio of high fat chocolate food arm entries on the last day of untreated training (shaded bars), $15 \mathrm{~min}$ after a vehicle injection (open bars), or SB334867 (filled bars). Treatment of SB334867 significantly reduces the preference for the high fat chocolate food arm $\left(^{*} p<0.05\right.$, ${ }^{* *} p<0.001$, repeated measures ANOVA). $\boldsymbol{D}$, Schematic of the cue-conditioning paradigm. Delivery of a sucrose pellet was contingent on a tone/light conditioned stimulus (CS+) during a daily 30 min session. $\boldsymbol{E}$, Conditioned-approach behavior, measured as the ratio of nosepokes during the $C S+$ to the nosepokes 30 s following $C S+$ presentation, was not different between rats having daily injections of vehicle (i.p., $n=11)$ or SB334867 (15 mg/kg, i.p., $n=11, p>0.05)$.

highly salient appetitive stimuli. In this experiment, foodrestricted rats were exposed to repeated, unpredictable footshocks for $30 \mathrm{~min}$ per day for $7 \mathrm{~d}$, a paradigm reported to produce stress in rats (Swiergiel et al., 2007) (Fig. 7A). As in previous electrophysiology experiments, VTA slices were taken $24 \mathrm{~h}$ after the last session. Despite an increase in NMDAR eEPSCs in VTA neurons of animals exposed to repeated footshock, the oxA/hcrt1(100 nM)-mediated potentiation of NMDAR eEPSCs did not significantly differ from that previously determined in naive, FR, or regular food self-administering rats $(125 \pm 7 \% ; n=10, p>$ 

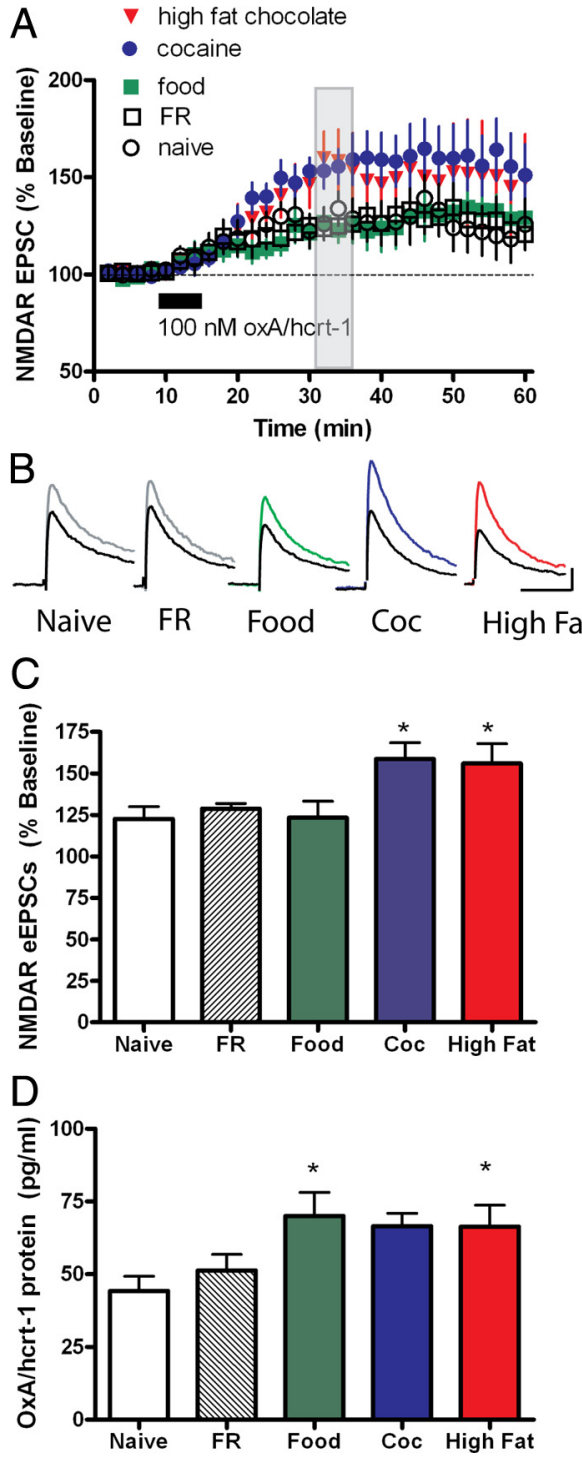

Figure 4. High fat chocolate food and cocaine self-administration selectively potentiates $0 \times A /$ hcrt-1-mediated NMDAR plasticity in VTA neurons. eEPSCs were recorded while neurons were voltage-clamped at $+40 \mathrm{mV}$ before and after a 5 -min bath application of oxA/hcrt-1 (100 nm). A, 0xA/hcrt-1 potentiated NMDAR eEPSCs in age-matched non-foodrestricted naive rats ( $n=7$; open circles). Food restriction did not alter $0 \times A /$ hcrt-1mediated potentiation of NMDAR eEPSC $\operatorname{in}$ FR rats ( $n=7$; open squares). $0 x A / \mathrm{hcrt}-1$ (100 $\mathrm{nm}, 5 \mathrm{~min}$ ) potentiated NMDAR eEPSC in regular food self-administering animals $(n=7$; green squares). Potentiation of NMDAR eEPSCs by $0 x A /$ hcrt- 1 was significantly enhanced after rats self-administered cocaine ( $n=9$; blue circles) or high fat chocolate food ( $n=$ 8 ; red triangles). $\boldsymbol{B}$, Example traces of NMDAR eEPSCs before (black trace) and 15 min after oxA/hcrt-1 in naive (gray), FR (gray), food (green), cocaine (blue), or high fat chocolate food (red) self-administering rats. Stimulus artifact has been removed for clarity. Calibration: 50 ms, 50 pA. C, Maximal oxA/hcrt-1-mediated potentiation of NMDAR eEPSCs is significantly greater in cocaine and high fat chocolate food self-administering rats compared with naive, FR and regular food self-administering rats $(p<0.05)$. Each bar represents the mean and SEM of EPSCs over a period of $2 \mathrm{~min}, 15 \mathrm{~min}$ after a $5 \mathrm{~min}$ application of oxA/hcrt-1 (100 nm) as shown by the shaded bar in $\boldsymbol{A}$. One-way ANOVA with a Bonferroni correction for multiple comparisons was used. D, The lateral hypothalamus was taken from horizontal midbrain slices used for electrophysiology $20-24 \mathrm{~h}$ after the final self-administration session. The tissue was "snap frozen" and processed later using a radio-immuno assay for determination of $0 \mathrm{xA} / \mathrm{hcrt}-1$ protein concentrations from naive ( $n=11$, open bar), FR ( $n=6$; shaded bar), regular food ( $n=9$; green bar), cocaine ( $n=$ 9; blue bar) or high-fat chocolate food ( $n=12$; red bar) self-administering rats. There were no significant differences between each group ( $p>0.05$, one-way ANOVA). Each bar represents the mean and SEM.
0.05) (Fig. 7B). Furthermore, the oxA/hcrt-1-mediated presynaptic effect on VTA neurons was not observed following footshock. OxA/hcrt-1 (100 nM) did not significantly alter mEPSC amplitude (before: $12 \pm 1 \mathrm{pA}$ vs after: $12 \pm 0.7 \mathrm{pA}, n=8, p>$ 0.05 ) (Fig. $7 C_{i}$ ) or frequency (before: $1.2 \pm 0.3 \mathrm{~Hz}$ vs after: $1.2 \pm$ $0.3 \mathrm{~Hz}, n=8, p>0.05$ ) (Fig. $7 C_{i i}$ ). These results suggest that, in contrast to data reported above for positive reinforcement, an aversive, arousing stimulus such as unpredictable footshock stress does not potentiate oxA/hcrt-1-mediated excitatory synaptic transmission in VTA dopamine neurons.

\section{Discussion}

In the present study, the ox/hcrt-1R antagonist, SB334867 decreased PR lever pressing selectively for cocaine and HF, but had no effect on PR responding for RF. Additionally, SB334867 reduced the effort required to obtain $\mathrm{HF}$ in an effort-based decision making task. Consistent with these findings, oxA/hcrt-1-mediated plasticity in the VTA was enhanced in animals self-administering cocaine or HF, but not RF. This increase in oxA/hcrt-1-mediated excitatory synaptic transmission observed in cocaine and HF groups was likely due to increased presynaptic signaling of ox/ hcrt-1Rs and enhanced glutamate release. These experiments demonstrate a role for oxA/hcrt-1-mediated signaling in motivation specifically for positive reinforcers of high salience, such as cocaine or HF pellets.

SB334867 has at least 50-fold selectivity over ox/hcrt-1Rs than $\mathrm{ox} / \mathrm{hcrt}$-2Rs and a wide range of other G-protein-coupled receptors (Porter et al., 2001). This antagonist can penetrate the bloodbrain-barrier and mediate behavioral effects following systemic administration. For example, an i.p. dose of $10 \mathrm{mg} / \mathrm{kg}$ yielded a brain concentration of $3.4 \mu \mathrm{M} 2 \mathrm{~h}$ post administration and could block oxA/hcrt-1-induced grooming or feeding (Porter et al., 2001). We have observed inhibition of oxA/hcrt-1mediated NMDAR currents of VTA dopamine neurons by 1 $\mu \mathrm{M}$ SB334867 in midbrain slices (Borgland et al., 2006), and thus we predict that SB334867 reduces work for reinforcers via antagonism of ox/hcrt-1Rs.

Dopamine action in the nucleus accumbens has been implicated in behavioral activation and effort-related processes, both components of motivated behavior (Salamone et al., 2007; Floresco et al., 2008). Our data indicates that ox/hcrt-1R-mediated signaling is important for enabling animals to overcome effort-related costs to obtain highly salient reinforcers. Systemic administration of SB334867 precludes precise determination of the specific neural regions where ox/hcrt-1 activity may mediate these effects. Nonetheless, our data reveal that oxA/hcrt-1 selectively potentiated presynaptic inputs into the VTA of animals self-administrating highly salient reinforcers. Thus, is it reasonable to propose that SB334867 may have reduced the actions of oxA/hcrt-1 on VTA neural activity, leading to attenuated dopamine activity in the nucleus accumbens. It is important to note that ox/hcrt-1Rs are expressed in other neurons of the mesocorticolimbic circuit underlying motivated behavior (Trivedi et al., 1998); therefore, future experiments may determine the exact locus of SB334867 action in reducing motivated behavior.

Our findings complement other studies showing that SB334867 also reduces PR responding for nicotine (Hollander et al., 2008), decreases the positive incentive properties of sexual behavior (Muschamp et al., 2007), and that centrally administered oxA/ hcrt-1 augments the breakpoint for sucrose pellets (Thorpe et al., 2005). Additionally, a selective increase in consumption of HF versus RF has been observed after oxA/hcrt-1 administration (Clegg et al., 2002; Zheng et al., 2007). OxA/hcrt-1 can augment 


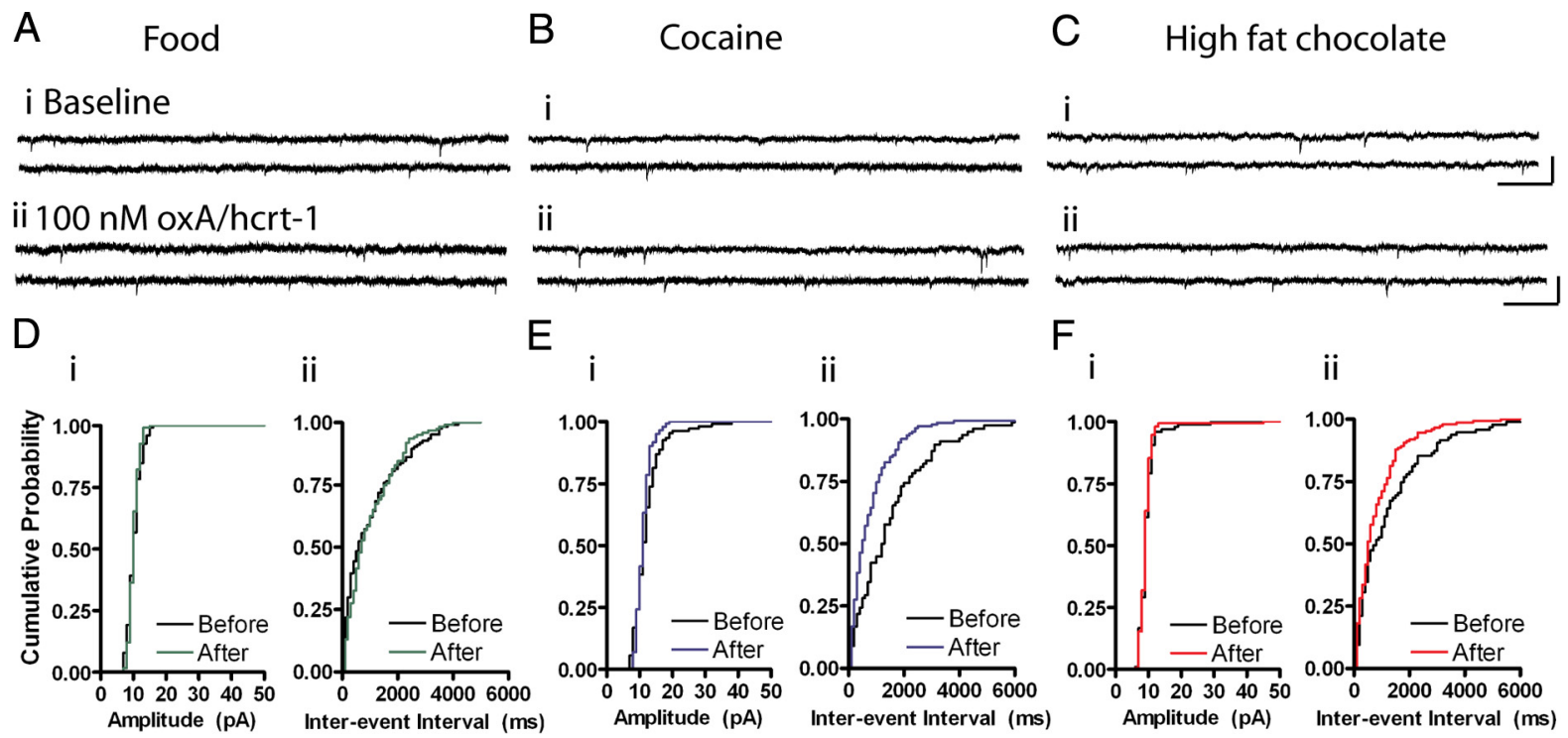

Figure 5. Cocaine or high fat self-administration increases presynaptic oxA/hcrt-1 signaling. $\mathrm{mEPSC}$ s were recorded while VTA neurons were voltage-clamped at $-70 \mathrm{mV}$ before and $10 \mathrm{~min}$ after a 5 min bath application of oxA/hcrt- 1 (100 nM). $A-C$, Example recordings of AMPAR mEPSCs before (i) and $15 \mathrm{~min}$ after (ii) oxA/hcrt- 1 application in VTA slices of rats self-administering ( $\boldsymbol{A}$ ) regular food, (B) cocaine, or ( $\boldsymbol{C}$ ) high-fat chocolate food. Calibrations: 50 pA, 200 ms. D, AMPAR mEPSC amplitude (i) or frequency (ii) was not significantly different after oxA/hcrt-1 application to VTA neurons from regular food self-administering rats $(n=8, p>0.05)$. $\boldsymbol{E}, \boldsymbol{F}$, In contrast, $\mathrm{mEPSC}$ frequency (iii), but not amplitude (i), was significantly increased after oxA/hcrt-1 application in $(\boldsymbol{E})$ cocaine $(n=8, p<0.05)$ or $(\boldsymbol{F})$ high fat chocolate food self-administering rats $(n=6, p<0.05)$. Each bar represents the mean and SEM. Paired t tests. $\boldsymbol{D}-\boldsymbol{F}$, Cumulative probability plots of amplitude (i) or interevent interval (ii) before (black) and after (colored) oxA/hcrt-1 application in example VTA neurons from $(\boldsymbol{D})$ regular food, $(\boldsymbol{E})$ cocaine, or $(\boldsymbol{F})$ high fat chocolate food self-administering rats.

feeding during altered motivational states such as following $24 \mathrm{~h}$ food deprivation, restricted feeding, or presentation of a highly palatable diet (Clegg et al., 2002; Szekely et al., 2002; Zheng et al., 2007; Nair et al., 2008). Here, we observed that ox/hcrt-1R sig-
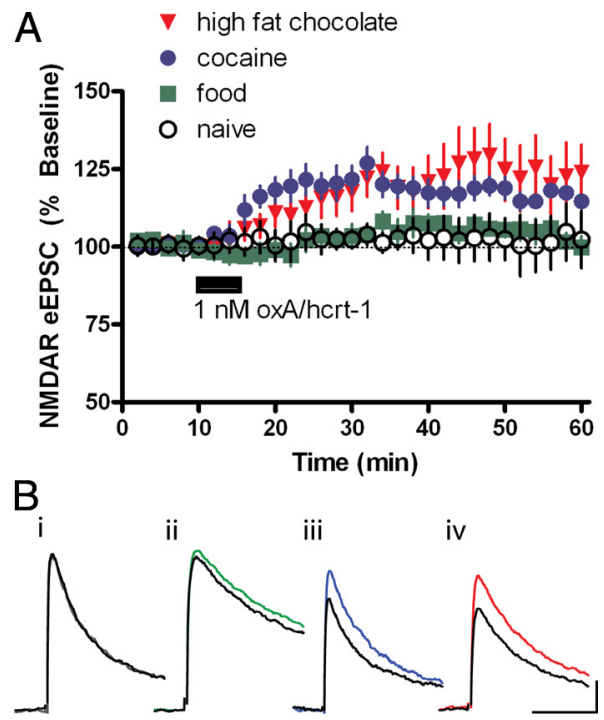

Figure 6. Orexin/hypocretin receptor signaling is increased after cocaine or high fat chocolate food self-administration. Rats self-administered regular food, cocaine or high fat chocolate food pellets on a fixed ratio 1 schedule. Horizontal midbrain sections were prepared $24 \mathrm{~h}$ after the last session. eEPSCs were recorded while neurons were voltage-clamped at $+40 \mathrm{mV}$ before and after a 5-min bath application of oxA/hcrt-1 (1 nm). Peak NMDAR current was measured 20 ms after the artifact. $A$, Application of $1 \mathrm{~nm}$ 0xA/hcrt-1 $(5 \mathrm{~min})$ did not potentiate NMDAR eEPSCs in VTA neurons of naive (open circles, $n=7$ ) or regular food self-administering rats (green squares, $n=8$ ). However, in rats self-administering cocaine (blue circles, $n=7$ ) or high fat chocolate food (red triangles, $n=8$ ), $1 \mathrm{~nm}$ oxA/hcrt-1 significantly potentiated NMDAR eEPSCs. $B$, Example traces of eEPSCs evoked at $+40 \mathrm{mV}$ before (black) and $10 \mathrm{~min}$ after (colored) oxA/hcrt-1 (1 nм, $5 \mathrm{~min}$ ) from naive rats (i, gray) or rats self-administering regular food (ii, green), cocaine (iii, blue) or high fat chocolate food (iv, red). Stimulus artifacts have been removed for clarity. Calibrations: $50 \mathrm{pA}, 50 \mathrm{~ms}$. naling was important for motivation for both cocaine and $\mathrm{HF}$ self-administration as well as effort to obtain HF pellets. Together, these data suggest that ox/hcrt-1R signaling underlies heightened motivation to obtain salient reinforcers.

The SB334867-induced reduction in responding for cocaine or HF could be due to disruption in memory for the reinforcer as the duration between reinforcer deliveries increased using the PR schedule (Akbari et al., 2007, 2008). However, this is unlikely because lever-pressing for RF pellets was maintained with SB33486. These data also suggest that the effects of SB334867 on responding for $\mathrm{HF}$ are unlikely to be attributable to satiety. Additionally, SB334867 did not affect the consumption of freely available RF or HF pellets in contrast with other reports showing that SB334867 reduces the hyperphagic response to i.c.v. oxA/ hcrt-1 in fasted rats free feeding on regular chow (Haynes et al., 2000) or in nonrestricted animals consuming highly palatable foods (Rodgers et al., 2001). This discrepancy may be because the effect of SB334867 on feeding was only observed at higher doses $(30 \mathrm{mg} / \mathrm{kg})$ which also affected grooming, sniffing, rearing, and locomotion (Rodgers et al., 2001). Thus, the $10 \mathrm{mg} / \mathrm{kg}$ dose of SB334867 used in the present study may induce a selective effect on motivated responding for highly salient reinforcers while leaving more general processes relative to hunger and satiety relatively intact.

$\mathrm{Ox} /$ hcrt-1R signaling has been implicated in the ability of food-associated conditioned stimuli to induce reward seeking behaviors, given that SB334867 impairs place preference for morphine or food (Harris et al., 2005, 2007; Narita et al., 2006) and cue-induced reinstatement of ethanol- or cocaine-seeking behavior (Lawrence et al., 2006; Smith et al., 2009). Yet, we observed that SB334867 did not affect the acquisition of conditionedapproach behavior for sucrose. Thus, the effects of SB334867 reported here do not appear to be mediated by a disruption in associative learning about reward-related stimuli.

Internal metabolic drive-state has been hypothesized to activate orexin/hypocretin neurons (Thorpe et al., 2003; Yamanaka 

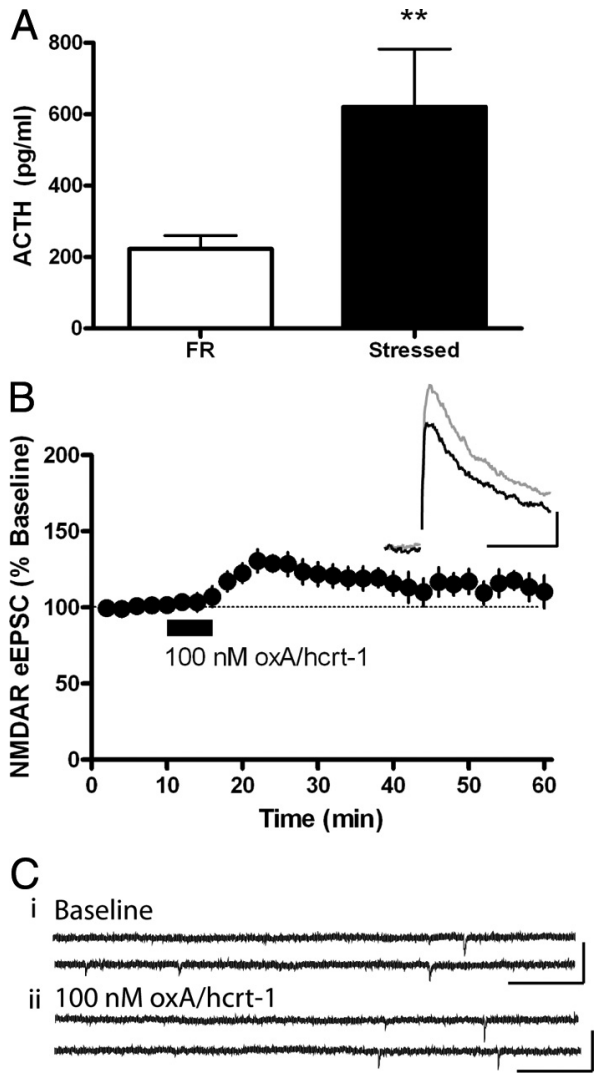

Figure 7. Footshock stress does not potentiate oxA/hcrt-1-mediated excitatory synaptic transmission in VTA neurons. Rats received 30 min of random footshocks for 7 consecutive days. Cardiac blood was drawn from FR $(n=6)$ or stressed $(n=6)$ rats immediately before aCSF perfusion and decapitation $24 \mathrm{~h}$ after the final footshock session. $A$, ACTH levels in stressed rats (filled bar) were significantly increased compared with FR controls (open bar; $p<0.05$ ). Bars represent mean and SEM. Unpaired Student's t test. Evoked EPSC $s$ were recorded while neurons were voltage clamped at $+40 \mathrm{mV}$ before and after a $5 \mathrm{~min}$ bath application of oxA/hcrt-1 (100 nm). $\boldsymbol{B}$, NMDAR eEPSCS of VTA neurons were potentiated by $100 \mathrm{~nm}$ oxA/hcrt- 1 in stressed rats ( $n=10$ ). Inset is an example eEPSC evoked at $+40 \mathrm{mV}$ before (black) and $10 \mathrm{~min}$ after (purple) a 5 min application of oxA/hcrt-1. Calibration: $50 \mathrm{pA}, 50 \mathrm{~ms}$. C, mEPSCs were recorded while VTA neurons were voltage clamped at $-70 \mathrm{mV}$ before and $10 \mathrm{~min}$ after a 5 -min bath application of oxA/hcrt-1 (100 nM). Example recordings of AMPAR mEPSCs before (i) and $15 \mathrm{~min}$ after (ii) oxA/hcrt-1 application in VTA slices of stressed rats. AMPAR mEPSC amplitude or frequency was not significantly different after oxA/hcrt- 1 application to VTA neurons of stressed rats ( $n=8$, $p>0.05$ ). Each bar represents the mean and SEM of 8 neurons from at least 3 rats. Paired $t$ tests.

et al., 2003; Burdakov and Alexopoulos, 2005), which may promote foraging behavior. For example, the intrinsic firing rate of orexin/hypocretin neurons can be altered by fluctuations in the levels of circulating nutrients and appetiteregulating hormones (Yamanaka et al., 2003; Burdakov and Alexopoulos, 2005). Orexin/hypocretin-mediated activation of catecholaminergic regions may promote locomotor activity in association with motivated behaviors (Wisor et al., 2001; Yamanaka et al., 2003). The present data support this hypothesis by suggesting that $\mathrm{ox} / \mathrm{hcrt}-1 \mathrm{R}$ signaling enhances motivation for highly salient reinforcers.

In our PR experiments, we observed lower breakpoints for cocaine versus RF or HF, which is likely attributable to the limitation of cocaine intake due to its peripheral effects (increased heart rate and blood pressure). Surprisingly, there were no differences in breakpoints in responding for HF or RF under control conditions, although ox/hcrt-1R blockade selectively reduced breakpoints in rats responding for HF or cocaine, but not RF. It is important to note that a number of factors can affect breakpoints and the lack of difference between animals working for RF or HF does not necessarily imply that the HF reinforcer was of equal salience to RF. Indeed, in a separate experiment, we demonstrated that when given the choice between the two reinforcers, rats are willing to expend more effort to climb a barrier to obtain HF pellets rather than choose freely available RF. Thus, the relative breakpoint among the different groups of vehicle-treated rats may not necessarily reflect the relative salience of the reinforcer, as has been discussed recently by Salamone et al. (2009). Because $\mathrm{ox} /$ hcrt-1R antagonism reduced a preference for the barriered HF arm, as well as the breakpoints for cocaine or HF, but not RF, these data further support the notion that ox/hcrt-1Rs mediate responding for highly salient, but not all reinforcers.

OxA/hcrt-1 potentiates NMDAR currents in VTA dopamine neurons in juvenile rats via protein kinase $\mathrm{C}$-induced trafficking of postsynaptic NMDARs (Borgland et al., 2006). Similarly, oxA/ hcrt-1 potentiates NMDARs in adult rats and this plasticity was not influenced by mild food restriction. Interestingly, selfadministration of cocaine or HF, but not RF, enhanced the oxA/ hcrt-1-mediated potentiation of NMDARs via an additional presynaptic increase in glutamate release as indicated by an increase in AMPAR mEPSC frequency but not amplitude. Furthermore, cocaine or HF pellet self-administration resulted in an enhanced sensitivity to low concentrations of oxA/hcrt-1 suggesting that self-administration of these highly salient reinforcers resulted in an alteration of ox/hcrt-1R coupling, or an enhancement in the signal transduction pathway in presynaptic afferents to the VTA.

Orexin/hypocretin neurons are activated by corticotropin releasing factor (CRF) and other stressful stimuli (WinskySommerer et al., 2004; Boutrel and de Lecea, 2008). Stressful stimuli are also known to induce synaptic plasticity in the VTA in an NMDAR-dependent manner (Saal et al., 2003). Thus, we wanted to determine whether stress could potentiate oxA/hcrt-1mediated excitatory synaptic transmission in the VTA in similar manner to that observed after cocaine or HF self-administration. After chronic footshock, there was no difference in oxA/hcrt-1 potentiated NMDAR currents compared with naive rats and no additional presynaptic effect as was observed with cocaine or HF self-administering rats. While it is possible that stress occludes the postsynaptic effect of oxA/hcrt-1 because both CRF and oxA/ hcrt-1 induce trafficking of NMDARs through similar mechanisms (Ungless et al., 2003; Borgland et al., 2006), it is unlikely that an oxA/hcrt-1-medidated presynaptic effect would be occluded by stress. CRF and oxA/hcrt-1 likely act on separate neural inputs into the VTA as an ox/hcrt-1R antagonist did not block CRF-dependent footshock-induced reinstatement of cocaine seeking or associated glutamate or dopamine release in the VTA (Wang et al., 2009). Furthermore, reward and arousal functions of oxA/hcrt-1 have been hypothesized to be represented in different neural circuits whereby orexin/hypocretin neurons that are activated by stimuli associated with positive reinforcement are located in the lateral hypothalamus and project to the VTA, whereas orexin/hypocretin neurons of the dorsal medial hypothalamus and perifornical area mediate arousal and response to stress (Harris and Aston-Jones, 2006).

In conclusion, these data support a role for oxA/hcrt-1 in motivation for highly salient positive reinforcers. Enhanced oxA/ hcrt-1-mediated potentiation of glutamatergic synaptic transmission in the VTA, only observed with highly salient appetitive reinforcers, may underlie the motivational drive to selectively seek cocaine or highly salient foods. We hypothesize that oxA/ hort-1 transmission in the VTA strengthens reinforcing proper- 
ties of highly salient stimuli, thus promoting motivation selectively for these rewards. These data contribute to our understanding of the role of orexin/hypocretin transmission in both normal reward processing for highly palatable foods as well as pathological reward processing for addictive drugs like cocaine.

\section{References}

Adamantidis AR, Zhang F, Aravanis AM, Deisseroth K, de Lecea L (2007) Neural substrates of awakening probed with optogenetic control of hypocretin neurons. Nature 450:420-424.

Akbari E, Naghdi N, Motamedi F (2007) The selective orexin 1 receptor antagonist SB-334867-A impairs acquisition and consolidation but not retrieval of spatial memory in Morris water maze. Peptides 28:650-656.

Akbari E, Motamedi F, Naghdi N, Noorbakhshnia M (2008) The effect of antagonization of orexin 1 receptors in CA1 and dentate gyrus regions on memory processing in passive avoidance task. Behav Brain Res 187: $172-177$.

Aston-Jones G, Smith RJ, Moorman DE, Richardson KA (2009) Role of lateral hypothalamic orexin neurons in reward processing and addiction. Neuropharmacology 56 [Suppl 1]:112-121.

Balcita-Pedicino JJ, Sesack SR (2007) Orexin axons in the rat ventral tegmental area synapse infrequently onto dopamine and gamma-aminobutyric acid neurons. J Comp Neurol 503:668-684.

Berridge KC (2007) The debate over dopamine's role in reward: the case for incentive salience. Psychopharmacology 191:391-431.

Borgland SL, Malenka RC, Bonci A (2004) Acute and chronic cocaineinduced potentiation of synaptic strength in the VTA: electrophysiologi$\mathrm{cal}$ and behavioral correlates in individual rats. J Neurosci 24:7482-7490.

Borgland SL, Taha SA, Sarti F, Fields HL, Bonci A (2006) Orexin A in the VTA is critical for the induction of synaptic plasticity and behavioral sensitization to cocaine. Neuron 49:589-601.

Boutrel B, de Lecea L (2008) Addiction and arousal, the hypocretin connection. Physiol Behav 93:947-951.

Boutrel B, Kenny PJ, Specio SE, Martin-Fardon R, Markou A, Koob GF, de Lecea L (2006) Role for hypocretin in mediating stress-induced reinstatement of cocaine-seeking behavior. Proc Natl Acad Sci U S A 102:19168-19173.

Burdakov D, Alexopoulos H (2005) Metabolic state signalling through central hypocretin/orexin neurons. J Cell Mol Med 9:795-803.

Carelli RM, Ijames SG, Crumling AJ (2000) Evidence that separate neural circuits in the nucleus accumbens encode cocaine versus "natural" (water and food) reward. J Neurosci 20:4255-4266.

Clegg DJ, Air EL, Woods SC, Seeley RJ (2002) Eating elicited by orexin-a, but not melanin-concentrating hormone, is opioid mediated. Endocrinology 143:2995-3000.

de Lecea L, Kilduff TS, Peyron C, Gao X, Foye PE, Danielson PE, Fukuhara C, Battenberg EL, Gautvik VT, Bartless FS 2nd, Frankel WN, van den Pol AN, Bloom FE, Gautvik KM, Sutcliffe JG (1998) The hypocretins: hypothalamus-specific peptides with neuroexcitatory activity. Proc Natl Acad Sci U S A 95:322-327.

Floresco SB, Ghods-Sharifi S (2007) Amygdala-prefrontal cortical circuitry regulates effort-based decision making. Cereb Cortex 17:251-260.

Floresco SB, St. Onge JR, Ghods-Sharifi S, Winstanley CA (2008) Corticolimbic-striatal circuits subserving different forms of cost-benefit decision making. Cogn Affect Behav Neurosci 8:375-389.

Harris GC, Aston-Jones G (2006) Arousal and reward: a dichotomy in orexin function. Trends Neurosci 29:571-577.

Harris GC, Wimmer M, Aston-Jones G (2005) A role for lateral hypothalamic orexin neurons in reward seeking. Nature 437:556-559.

Harris GC, Wimmer M, Randall-Thompson JF, Aston-Jones G (2007) Lateral hypothalamic orexin neurons are critically involved in learning to associate an environment with morphine reward. Behav Brain Res 183:43-51.

Haynes AC, Jackson B, Chapman H, Tadayyon M, Johns A, Porter RA, Arch JR (2000) A selective orexin-1 receptor antagonist reduces food consumption in male and female rats. Regul Pept 96:45-51.

Hollander JA, Lu Q, Cameron MD, Kamenecka TM, Kenny PJ (2008) Insular hypocretin transmission regulates nicotine reward. Proc Natl Acad Sci U S A 105:19480-19485.

Johnson SW, North RA (1992) Two types of neurone in the rat ventral tegmental area and their synaptic inputs. J Physiol 450:455-468.
Jones S, Bonci A (2005) Synaptic plasticity and drug addiction. Curr Opin Pharmacol 5:20-25.

Korotkova TM, Sergeeva OA, Eriksson KS, Haas HL, Brown RE (2003) Excitation of ventral tegmental area dopaminergic and nondopaminergic neurons by orexin/hypocretins. J Neurosci 23:7-11.

Lacey MG, Mercuri NB, North RA (1990) Actions of cocaine on rat dopaminergic neurons in vitro. Br J Pharmacol 99:731-735.

Lawrence AJ, Cowen MS, Yang HJ, Chen F, Oldfield B (2006) The orexin system regulates alcohol-seeking in rats. Br J Pharmacol 148:752-759.

Lowry OH, Rosebrough NJ, Farr AL, Randall RJ (1951) Protein measurement with the folin phenol reagent. J Biol Chem 193:265-275.

Mileykovskiy BY, Kiyashchenko LI, Siegel JM (2005) Behavioral correlates of activity in identified hypocretin/orexin neurons. Neuron 46:787-798.

Muschamps JW, Dominguez JM, Sato SM, Shen RY, Hull EM (2007) A role for hypocretin (orexin) in male sexual behaviour. J Neurosci 27:2837-2845.

Nair SG, Golden SA, Shaham Y (2008) Differential effects of the hypocretin 1 receptor antagonist SB334867 on high-fat food self-administration and reinstatement of food seeking in rats. Br J Pharmacol 154:1-11.

Narita M, Nagumo Y, Hashimoto S, Narita M, Khotib J, Miyatake M, Sakurai T, Yanagisawa M, Nakamachi T, Shioda S, Suzuki T (2006) Direct involvement of orexinergic systems in the activation of the mesolimbic dopamine pathway and related behaviors induced by morphine. J Neurosci 26:398-405.

Peyron C, Tighe DK, van den Pol AN, de Lecea L, Heller HC, Sutcliffe JG, Kilduff TS (1998) Neurons containing hypocretin (orexin) project to multiple neuronal systems. J Neurosci 18:9996-10015.

Porkka-Heiskanen T, Kalinchuk A, Alanko L, Huhtaniemi I, Stenberg D (2004) Orexin A and B levels in the hypothalamus of female rats: the effects of the estrous cycle and age. Eur J Endocrinol 150:737-742.

Porter RA, Chan WN, Coulton S, Johns A, Hadley MS, Widdowson K, Jerman JC, Brough SJ, Coldwell M, Smart D, Jewitt F, Jeffrey P, Austin N (2001) Effects of chronic footshock, restraint and corticotropin-releasing factor on freezing, ultrasonic vocalization and forced swim behavior in rats. Bioorg Med Chem Lett 11:1907-1910.

Richardson NR, Roberts DC (1996) Progressive ratio schedules in drug selfadministration studies in rats: a method to evaluate reinforcing efficacy. J Neurosci Methods 66:1-11.

Rodgers RJ, Halford JC, Nunes de Souza RL, Canto de Souza AL, Piper DC, Arch JR, Upton N, Porter RA, Johns A, Blundell JE (2001) SB-334867, a selective orexin-1 receptor antagonist, enhances behavioural satiety and blocks the hyperphagic effect of orexin-A in rats. Eur J Neurosci 13:1444-1452.

Saal D, Dong Y, Bonci A, Malenka RC (2003) Drugs of abuse and stress trigger a common synaptic adaptation in dopamine neurons. Neuron 37:577-582.

Sakurai T, Amemiya A, Ishii M, Matsuzaki I, Chemelli RM, Tanaka H, Williams SC, Richardson JA, Kozlowski GP, Wilson S, Arch JR, Buckingham RE, Haynes AC, Carr SA, Annan RS, McNulty DE, Liu WS, Terrett JA, Elshourbagy NA, Bergsma DJ, Yanagisawa M (1998) Orexin and orexin receptors: a family of hypothalamic neuropeptides and G proteincoupled receptors that regulate feeding behavior. Cell 92:573-585.

Salamone JD, Correa M, Farrar A, Mingote SM (2007) Effort-related functions of nucleus accumbens dopamine and associated forebrain circuits. Psychopharmacology 191:461-482.

Salamone JD, Correa M, Farrar M, Nunes EJ, Pardo M (2009) Dopamine, behavioral economics, and effort. Front Behav Neurosci. Advance online publication. Retrieved March 13, 2009. doi:10.3389/neuro.08.013.2009.

Smith RJ, See RE, Aston-Jones G (2009) Orexin/hypocretin signaling at the OX1 receptor regulates cue-elicited cocaine seeking. Eur J Neurosci 30:493-503.

Stuber GD, Klanker M, de Ridder B, Bowers MS, Joosten RN, Feenstra MG, Bonci A (2008) Reward predictive cues enhance excitatory synaptic strength onto midbrain dopamine neurons. Science 321:1690-1692.

Swiergiel AH, Zhou Y, Dunn AJ (2007) Effects of chronic footshock, restraint and corticotropin-releasing factor on freezing, ultrasonic vocalization and forced swim behavior in rats. Behav Brain Res 183:178-187.

Székely M, Pétervári E, Balaskó M, Hernádi I, Uzsoki B (2002) Effects of orexins on energy balance and thermoregulation. Regul Pept 104:47-53.

Thorpe AJ, Mullett MA, Wang C, Kotz CM (2003) Regional, metabolic and circadian specificity of LH orexin A. Am J Physiol Regul Integr Comp Physiol 284:R1409-R1417. 
Thorpe AJ, Cleary JP, Levine AS, Kotz CM (2005) Centrally administered orexin A increases motivation for sweet pellets in rats. Psychopharmacology 182:75-83.

Trivedi P, Yu H, MacNeil DJ, Van der Ploeg LH, Guan XM (1998) Distribution of orexin receptor mRNA in the rat brain. FEBS Lett 438:71-75.

Ungless MA, Whistler JL, Malenka RC, Bonci A (2001) Single cocaine exposure in vivo induces long-term potentiation in dopamine neurons. Nature 411:583-587.

Ungless MA, Singh V, Crowder TL, Yaka R, Ron D, Bonci A (2003) Corticotropin-releasing factor requires CRF binding protein to potentiate NMDA receptors via CRF receptor 2 in dopamine neurons. Neuron 39:401-407.

Vittoz NM, Berridge CW (2006) Hypocretin/orexin selectively increases dopamine efflux within the prefrontal cortex: involvement of the ventral tegmental area. Neuropsychopharmacology 31:384-395.

Vittoz NM, Schmeichel B, Berridge CW (2008) Hypocretin/orexin preferentially activates caudomedial ventral tegmental area dopamine neurons. Eur J Neurosci 28:1629-1640.

Wang B, You Z, Wise RA (2009) Independence of VTA orexin and CRF mechanisms in the reinstatement of cocaine-seeking. Biol Psychiatry, in press.

Winsky-Sommerer R, Yamanaka A, Diano S, Borok E, Roberts AJ, Sakurai T, Kilduff TS, Horvath TL, de Lecea L (2004) Interaction between the corticotropin-releasing factor system and hypocretins (orexins): a novel circuit mediating stress response. J Neurosci 24:11439-11448.

Wisor JP, Nishino S, Sora I, Uhl GH, Mignot E, Edgar DM (2001) Dopaminergic role in stimulant-induced wakefulness. J Neurosci 21:1787-1794.

Yamanaka A, Beuckmann CT, Willie JT, Hara J, Tsujino N, Mieda M, Tominaga M, Yagami K, Sugiyama F, Goto K, Yanagisawa M, Sakurai T (2003) Hypothalamic orexin neurons regulate arousal according to energy balance in mice. Neuron 38:701-713.

Zhang M, Balmadrid C, Kelley AE (2003) Nucleus accumbens opioid, GABaergic, and dopaminergic modulation of palatable food motivation: contrasting effects revealed by a progressive ratio study in the rat. Behav Neurosci 117:202-211.

Zheng H, Patterson LM, Berthoud HR (2007) Orexin signaling in the ventral tegmental area is required for high fat appetite induced by opioid stimulation of the nucleus accumbens. J Neurosci 27:11075-11082. 